\title{
Gas lasers excited by a pulsed inductive discharge
}

The XII International Conference on Laser Optics (LO-2006), St.Petersburg, Russia, June 26 to 30, 2006

\author{
Alexander M. Razhev*a , Vahram M. Mekhitarian ${ }^{\mathrm{b}}$, Dmitry S. Churkin ${ }^{\mathrm{a}}$, Andrey A. Zhupikov ${ }^{\mathrm{a}}$, \\ ${ }^{a}$ Institute of Laser Physics SD RAS, Lavrentyev ave. 13/3, 630090, Novosibirsk, Russia; \\ ${ }^{\mathrm{b}}$ Institute of Physical Researches NAS RA, Ashtarak-2, 378410, Armenia
}

\begin{abstract}
A pulsed transverse inductive discharge (PTID) is used to population inversion formation on the transitions in atoms and molecules. Excitation system producing an inductive discharge in gases under a pressure within a range from 0,1 to 1,0 atm has been created. The experimental study of spectral and temporal parameters of stimulated emission for active media on the $\mathrm{Ne}(585.3 \mathrm{~nm}), \mathrm{He}(587.5,667.8 \mathrm{~nm}), \mathrm{Ar}(750.4 \mathrm{~nm})$ atoms and $\mathrm{XeF}^{*}(353 \mathrm{~nm}), \mathrm{XeCl} *(308 \mathrm{~nm})$ and $\mathrm{KrF}^{*}$ $(248 \mathrm{~nm})$, molecules excited by an inductive transverse discharge was performed. Lasing on the transitions of fluorine atoms and nitrogen molecules are reported. The results of the experimental study of spectral, temporal, and energy parameters of fluorine (FI) and nitrogen inductive lasers are presented.
\end{abstract}

Keywords: pulsed transverse inductive discharge, laser emission

\section{INTRODUCTION}

The RF induction excitation of continuous-wave lasing was reported in papers ${ }^{1-2}$. Continuous-wave (cw) lasing on the transitions in atomic argon ions in the green spectral range under excitation by longitudinal inductive RF discharge was obtained in these papers. The possibility of developing a laser excited by a pulsed inductive discharge was discussed in paper $^{3}$.

In this work, a method for laser active media excitation by a pulsed inductive transverse discharge is proposed and experimentally realized for the first time in order to obtain the lasing on electron transitions in an atoms and molecules in gases. It is important fact that the pulse repetition rate must be several hertz and higher. That means that all processes of discharge formation, creation of population inversion, amplification, absorption and quenching must occur during each pulse irrespective of the past history of the preceding pulse.

It is well known that a pulsed transverse electric discharge as an excitation method has a number of advantages over pulsed longitudinal or continuous discharges. Here the transverse discharge is determined as an electric discharge in which the path of a current flow is perpendicular to the optical axis. Such a discharge ensures the lasing on a very large number of transitions in atoms, molecules, and their ions due to the realization of various (including new) mechanisms of population inversion formation in a wide range of pressures, including atmospheric one. This lasing is achieved due to the fact that in transverse discharge a much higher level of the excitation pulsed power can be reached. It leads to a lasing on the variety of transitions including those for which no gain will be obtained by using the other discharge types. As a result, lasers with high output radiation energy and a high efficiency (1-10\%) can be developed.

In contrast to conventional pulsed longitudinal and transverse discharges, a pulsed inductive transverse discharge (PTID) is formed due to the magnetic field inductance produced by the excitation system without any electrodes into the active medium. An appropriate choice of the tube material may ensure the active medium purity and to realize a high laser service life.

The formation of such a discharge is not accompanied by the appearance of cathode spots on the electrode surfaces, which are responsible for the discharge instability and contraction. And as consequence it leads to decrease of the discharge homogeneity, a gas mixture contamination, quenching the lasing and a limitation of the pulse repetition rate.

*razhev@laser.nsc.ru; phone 7383 3306214; fax 7383 3332067; www.laser.nsc.ru

Proc. SPIE Vol. 6611, Laser Optics 2006: High-Power Gas Lasers, 66110G p. 1-11, April 2007 
The use of the pulsed inductive discharge is a promising method for excitation not only gas lasers but also metal vapor lasers. Additionally, this method can be used to produce plasma for obtaining radiation (including induced one) in any spectral range, especially for the range from 10 to $150 \mathrm{~nm}$ having a considerable interest for microelectronics and photolithography.

The excimer lasers are widely known and described in a lot of papers. These lasers have the radiation wavelengths in the UV range of spectra 193-353 $\mathrm{nm}$ and are capable to obtain the radiation with output energy of $1.0 \mathrm{~J}$ with efficiency of several percent ${ }^{4-8}$. The typical excitation method for excimer lasers is a transverse discharge with a preionisation of the gas mixture. We think that from the laser physics point of view an obtaining the lasing on the transitions of excimer molecules pumped by pulsed inductive transverse discharge and the study of the inversion population producing mechanism into inductive discharge have the great interest.

Lasers on the atomic transitions of the rare gases $\mathrm{He}(706.5 \mathrm{~nm}), \mathrm{Ne}(585.3 \mathrm{~nm})$ and $\mathrm{Ar}(750.4 \mathrm{~nm})$ are widely described in the literature. Despite to the low efficiency they are very interesting from the point of view of the collision population mechanisms for a various electronic levels of rare gas atoms and inversion population producing on some transitions. One can emphasize some papers ${ }^{9-12}$ in which the $\mathrm{H}_{2}$ and $\mathrm{NF}_{3}$ additives were used to increase the lasing efficiency on the rare gases transitions. These additives provide the depopulation of the lower laser level and as a consequence the increase of the power and duration of a lasing. A transverse discharge was used as an excitation method. In this paper we have tried to use a pulsed inductive transverse discharge for active medium pumping.

There are many works ${ }^{13-24}$ on lasing on various transitions in fluorine atoms in the spectral range of 623-780 nm. Lasing was observed on 17 lines. However, owing to different excitation conditions, the spectra obtained in different works differ in the number of lines with various wavelengths. The population inversion on atomic fluorine transitions was created using two excitation methods, a longitudinal electric discharge ${ }^{13-17}$ in low-pressure (0.5-50 torr) helium mixtures with fluorinecontaining molecules and a transverse discharge ${ }^{18,20,21,23}$ ensuring lasing in the same mixtures in a wide pressure range up to $3 \mathrm{~atm}$. At a low pressures, $\mathrm{HF}, \mathrm{SF}_{6}, \mathrm{CF}_{4}, \mathrm{C}_{2} \mathrm{~F}_{6}, \mathrm{NF}_{3}$ and $\mathrm{F}_{2}$ molecules were used fluorine as a fluorine donors, while only $\mathrm{NF}_{3}$ and $\mathrm{F}_{2}$ molecules were used for high pressures due to the discharge homogeneity achieved using the UV preionisation of the discharge gap. In most available experiments, lasing on fluorine atomic transitions took place in the superluminescence regime. The laser pulse duration in various experiments varied from a few microseconds (longitudinal discharge) to tens of nanoseconds (transverse discharge). The gas mixture forming the active medium of the laser usually consisted of helium and admixtures of fluorine-containing molecules in the ratio of 30:1 to 1000:1. Helium was required for creating inversion on transitions in fluorine atoms via the excited states of helium atoms. The use of other rare gases (such as $\mathrm{Ne}$ or $\mathrm{Ar}$ ) as a buffer gas did not lead to lasing.

The first successful experiments on lasing on the electronic transition of fluorine atom under excitation of by PTID were reported in ${ }^{25}$ where first inductive FI laser was created.

As it follows from literature analysis, the pulsed transverse inductive discharge can be a promising excitation method of pumping for various gas active media. Therefore the main aim of this work was to forming the inversion population on the atomic and molecular transitions of different gases excited by a pulsed transverse inductive discharge and an achievement of the laser action.

\section{EXPERIMENTAL SETUP AND MEASUREMENT EQUIPMENT}

To produce a pulsed transverse inductive discharge we used two different excitation systems. One system was developed on the base of the excitation scheme of a $L C$-inverter type and it is described in detail in ${ }^{25}$. Another system is shown on the Figure 1. The system was developed on the base of a capacitor-transfer type excitation scheme with triggered spark gap $S G_{1}$ as a high voltage switch and with a peaking capacitance $C_{2}$. A self-triggered spark gap $S G_{2}$ was used into the excitation system as a current interrupter. The charging voltage $U_{c h}$ on the storage capacitor $C_{1}$ was varied from 20 to 27 $\mathrm{kV}$.

The inductor $L_{l}$ was a solenoid with length $40-60 \mathrm{~cm}$. The solenoid consisted of individual solenoids prepared in the form of parallel-connected sections wound on a glass tube into which gas mixtures were admitted. Each section contained 3 to 20 turns of a stranded insulated cable with a cross-section from 1.5 to $4 \mathrm{~mm}^{2}$. In experiments, the inductor 
parameter such as the total inductance and resistance were varied to ensure the required values of magnetic induction and the electric field strength in the tube and, hence, the inductive discharge parameters.

The experiments were carried out with glass tubes with an inner diameter of 1 to $4 \mathrm{~cm}$ and an active lengths $40-60 \mathrm{~cm}$. At the ends of the tube, adjusting units with plane-parallel quartz or $\mathrm{MgF}_{2}$ windows $W_{l}$ and $W_{2}$ were fixed. The windows were arranged perpendicularly to the tube axis and were used as sealing and optical cavity elements. On one side of the tube, a high-reflective plane $\mathrm{Al}$ or dielectric mirror $M$ was placed.

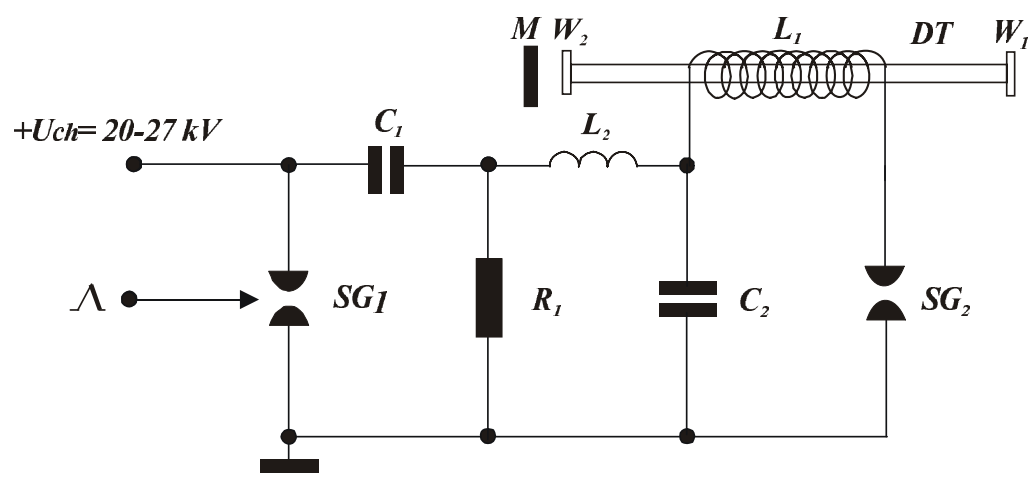

Fig. 1. Electric circuit of a laser excitation system by a pulsed inductive transverse discharge: $U$ - charging voltage; $S G_{1}$ - spark gap; $S G_{2}$ - un-triggered spark gap; $C_{1}=48 \mathrm{nF} ; C_{2}=27 \mathrm{nF} ; R_{1}=1,0 \mathrm{k} \Omega ; D T-$ discharge tube; $L_{1}$ - inductor; $L_{2}$ - charging inductance $150 \mu \mathrm{H} ; W_{1}, W_{2}$ - quartz or $\mathrm{MgF}_{2}$ windows;

$M$ - rear resonator mirror.

In experiments we studied the intensity and duration of radiation emitted by the inductive discharge in various gases, as well as its spectral and energy parameters under different excitation conditions. In our measurements, we used FEK-22 and FEK-15 coaxial photocells with high temporal resolutions, Tektronix TDS 220 oscilloscope, an Ocean Optics HR 2000 spectrometer, and an Ophir Optronics pyroelectric laser radiation energy meter (PE50-BB and PE50 detectors). The accuracy of measurements was $5 \%$ in all experiments.

\section{RESULTS AND DISCUSSION}

In experiments the pure rare gases $\mathrm{He}, \mathrm{Ne}, \mathrm{Ar}, \mathrm{N}_{2}$ and their mixtures with $\mathrm{H}_{2}, \mathrm{~F}_{2}$ and $\mathrm{NF}_{3}$, the $\mathrm{He}: \mathrm{Xe}(\mathrm{Kr}): \mathrm{F}_{2}(\mathrm{HCl}), \mathrm{KrF}$ mixtures were excited by a pulsed transverse inductive discharge. The various compositions of these gases under various pressures were used as gas active media. Gas mixtures flows in longitudinal direction of the tube with a low velocity.

It must be pointed out that in the case of excitation of an active medium inside a discharge tube by a pulsed transverse inductive discharge we deal with the new form of laser beam - a ring beam which is determines on the excitation conditions and a diameter of a tube.

\subsection{Stimulated emission on the transitions of rare gases ( $\mathrm{Ne}, \mathrm{Ar}$ and $\mathrm{He}$ )}

The spectral, temporal and amplitude parameters of the inductive discharge emission for pure rare gases $\mathrm{Ne}, \mathrm{Ar}, \mathrm{He}$ and their mixtures with additives of $\mathrm{H}_{2}$ and $\mathrm{NF}_{3}$ were studied. In the experiments it was obtained that the intensity and duration of emission depend on the sort and the total pressure of rare gas and an admixture. For all gases mentioned, the stimulated emission usually started at pressures 20-40 torr, it observed up to 140 torr and then it disappeared at higher pressure. 
The dependence of the radiation intensity (a) and pulse duration (b) on the total pressure for Ne: $\mathrm{NF}_{3}-9: 1$ mixture are shown on the Figure 2 and the temporal traces of the pulses of the spontaneous and stimulated emission for different pressures are shown on the Figure 3. As it is shown on the Figure 2a in the low pressures range 10-20 torr, the stimulated emission intensity increases rapidly, reaches the peak within 20-40 torr, and then decreases slowly up to 140 torr. The pulse duration (FWHM) at pressure of 10 torr was $200 \mathrm{~ns}$ and with an increase of the pressure, when the lasing mode was achieved, the pulse duration shortened up to $20 \pm 5$ ns (Figure 2 b).

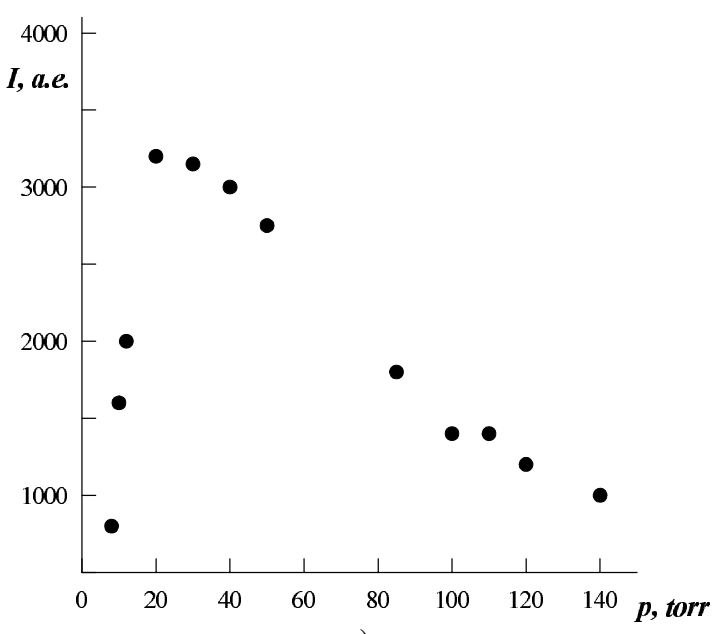

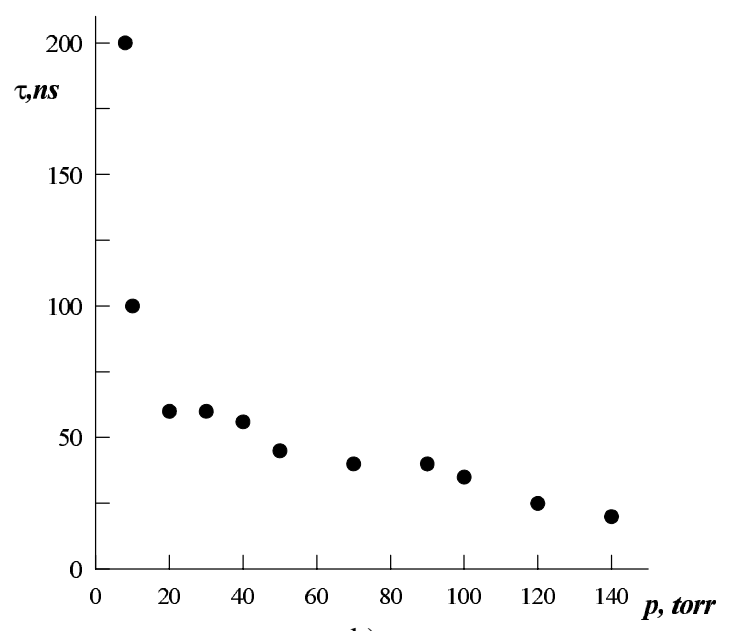

b)

Fig. 2. Dependence of the radiation intensity (a) and pulse duration $(\mathrm{FWHM})(\mathrm{b})$ on the total pressure for $\mathrm{Ne}: \mathrm{NF}_{3}-9: 1$ gas mixture, $U_{c h}=27 \mathrm{kV}$.
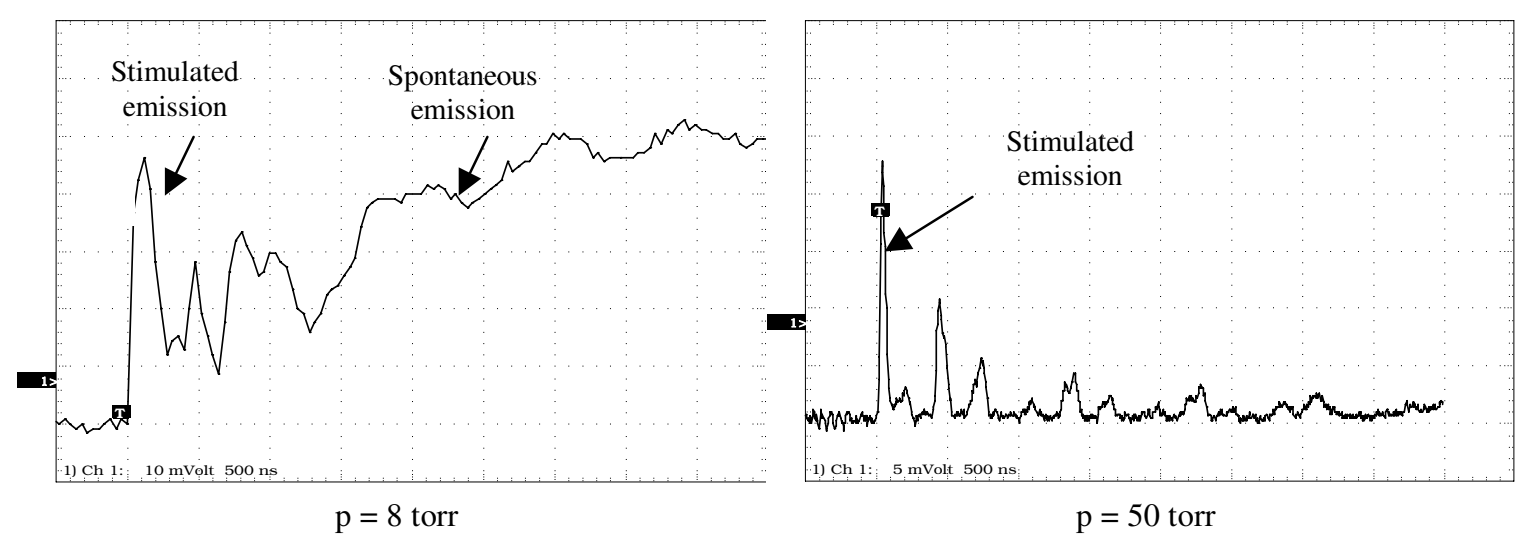

Fig. 3. Temporal traces of the spontaneous and stimulated emission pulses for $\mathrm{Ne}: \mathrm{NF}_{3}-9: 1$ mixture at different total pressure.

The same situation was observed for $\mathrm{Ar}$ and He rare gases in the experiments. The gain factor measured using a cavity loss method for the rare gas inductive lasers was $0.007-0.01 \mathrm{~cm}^{-1}$.

Analysis of the spectral composition of radiation for rare gas $(\mathrm{Ne}, \mathrm{Ar}$ an $\mathrm{He}$ ) shows that the number of spectral lines strongly depends on the gas medium composition and total pressure and that the using of $\mathrm{H}_{2}$ and $\mathrm{NF}_{3}$ additives is able to change the emission spectra. On the Figure 4-6 the spectra of pure Ne, Ar, He rare gases (a) and their mixtures with additives (b) are shown.

Proc. SPIE Vol. 6611, Laser Optics 2006: High-Power Gas Lasers, 66110G p. 1-11, April 2007 
The spectra of pure Ne gas consist of many lines in the region of 570-750 nm (Figure 4a). The addition of $\mathrm{H}_{2}$ or $\mathrm{NF}_{3}$ leads to the elimination of a most spectral lines and all emission intensity concentrates only in one line on the wavelength of $585.3 \mathrm{~nm}$ (Figure 4b).

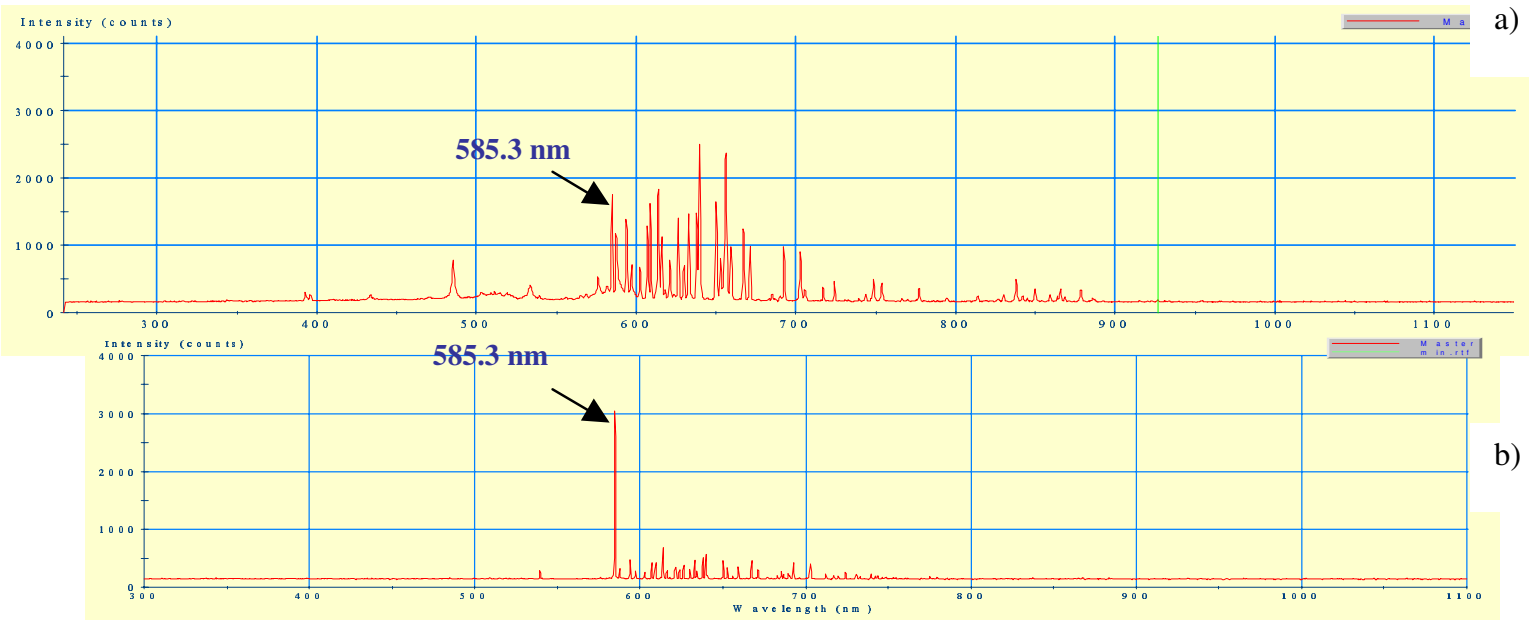

Fig. 4. Emission spectra of pure $\mathrm{Ne}$ gas under pressure $\mathrm{p}=100$ torr (a) and for $\mathrm{Ne}: \mathrm{NF}_{3}-9: 1$ mixture under pressure $\mathrm{p}=40$ torr or $\mathrm{Ne}: \mathrm{H}_{2}-3: 1$ mixture under pressure $\mathrm{p}=100$ torr $(\mathrm{b})$.

For pure Ar the spectra of consist of several lines in the region of 650-870 nm (Figure 5a). The addition of $\mathrm{H}_{2}$ or $\mathrm{NF}_{3}$ leads to significant increase of the emission intensity on the wavelength of $750.4 \mathrm{~nm}$ (Figure $5 \mathrm{~b}$ ).

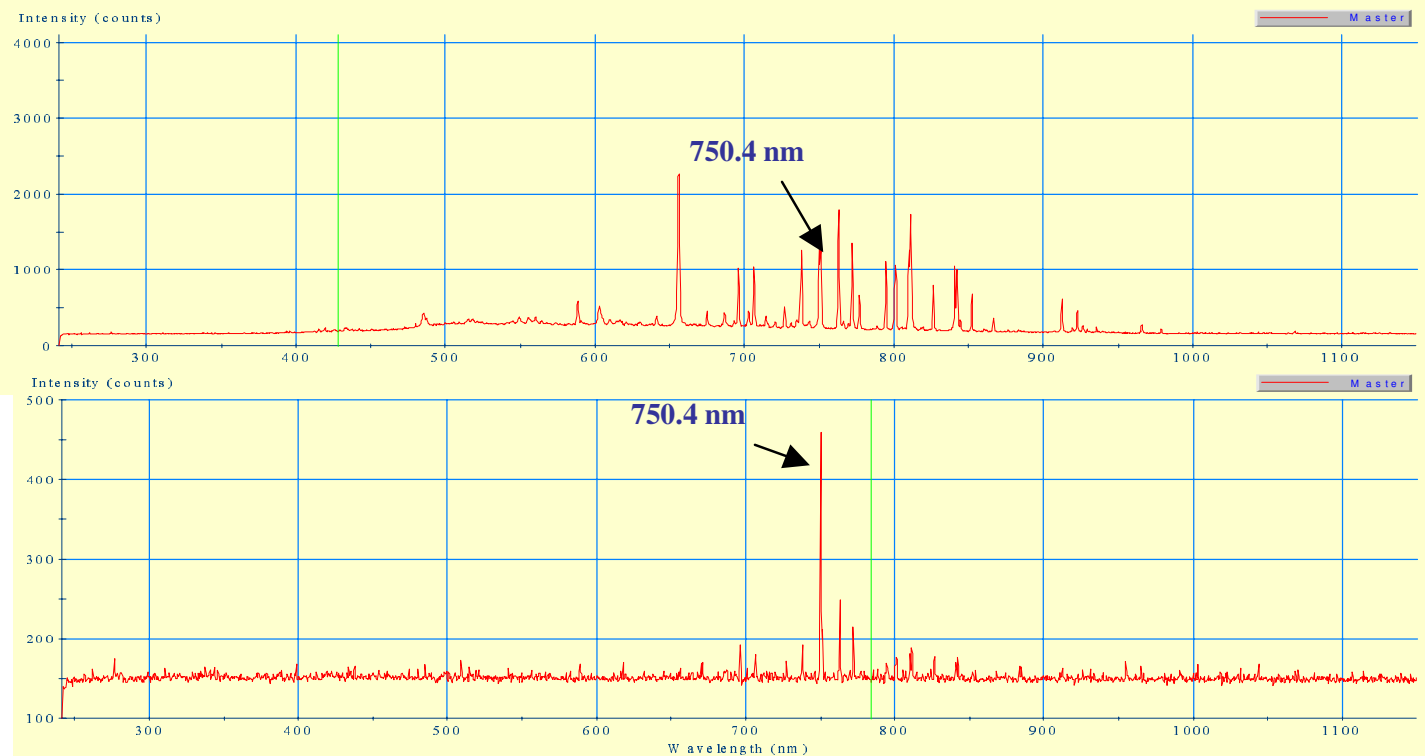

a)

Fig. 5. Emission spectra of pure Ar gas under pressure $\mathrm{p}=100$ torr (a) and for $\mathrm{Ar}: \mathrm{NF}_{3}-3: 1$ mixture under pressure $\mathrm{p}=20$ torr or Ar: $\mathrm{H}_{2}-3: 1$ mixture under $\mathrm{p}=50$ torr $(\mathrm{b})$.

Proc. SPIE Vol. 6611, Laser Optics 2006: High-Power Gas Lasers, 66110G p. 1-11, April 2007 66110G-5 
In the case of a pure He the spectra of consist of several lines in the region of 580-710 nm (Figure 6a). The addition $\mathrm{NF}_{3}$ leads to redistribution of the emission intensity and finally there is only two strong lines on the wavelengths of 587.5 and $706.5 \mathrm{~nm}$ (Figure 6b).
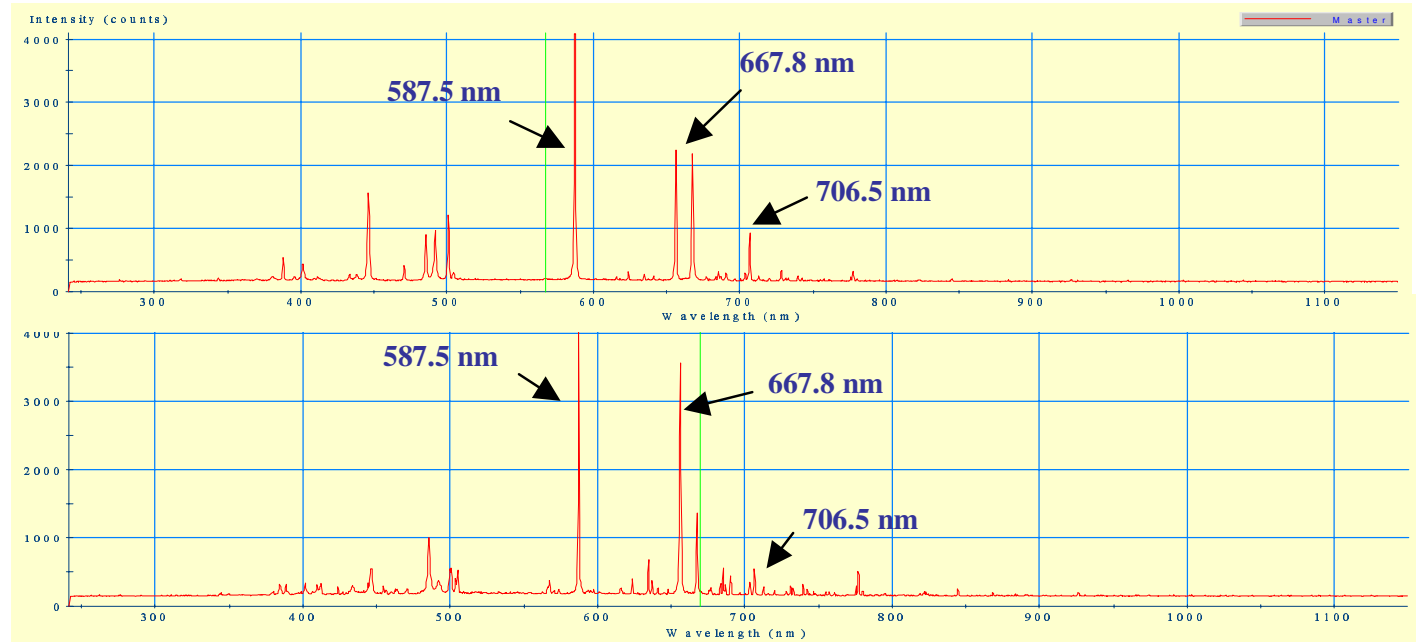

Fig. 6. Emission spectra of pure He under pressure $\mathrm{p}=100$ torr (a) and for $\mathrm{He}_{\mathrm{NF}}-10: 1$ mixture under pressure $\mathrm{p}=10$ torr (b).

\subsection{Stimulated emission on the $\mathrm{XeF}^{*}, \mathrm{XeCl}^{*}$ and $\mathrm{KrF}^{*}$ excimer molecules}

In these experiments we obtained the stimulated emission on the transitions of the excimer molecules having a radiation wavelengths in the UV region of spectra. To obtain the stimulated emission on the $\mathrm{XeF}^{*}(353 \mathrm{~nm})$ excimer molecules the energy and temporal parameters of emission for $\mathrm{He}: \mathrm{Xe}: \mathrm{F}_{2}$ gas mixtures in various compositions and under various pressures within the range 2-500 torr excited by PTID were studied. The temporal traces of the inductor voltage, spontaneous and stimulated emission pulses for He:Xe: $\mathrm{F}_{2^{-}}$97:2.5:0.5 mixture are shown on Figure 7.

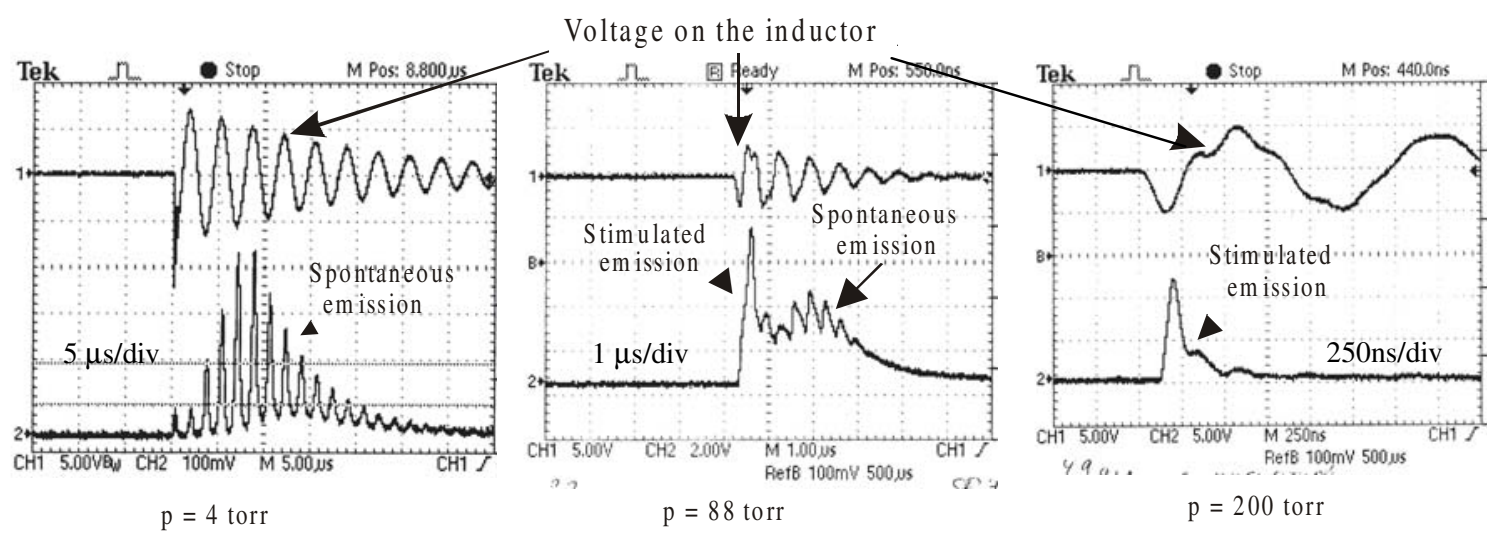

Fig. 7. The temporal traces of the inductor voltage, spontaneous and stimulated emission pulses at different pressures. $U_{c h}=24 \mathrm{kV}$.

The stimulated emission on the radiation wavelength of $353 \mathrm{~nm}$ started at 88 torr and was observed up to 450 torr. Maximum of the stimulated emission intensity was obtained for pressures of 300- 350 torr.

Proc. SPIE Vol. 6611, Laser Optics 2006: High-Power Gas Lasers, 66110G p. 1-11, April 2007 66110G-6 
The dependence of the stimulated emission pulse duration (FWHM) for $\mathrm{XeF}^{*}$ excimer molecules on the total pressure is shown on the Figure 8. As it seen from the figure the pulse duration for 40 torr pressure was $300 \mathrm{~ns}$ (FWHM) and with an increase of the pressure when the lasing mode was achieved the pulse duration shortened from $230 \mathrm{~ns}$ up to $30 \pm 5 \mathrm{~ns}$.

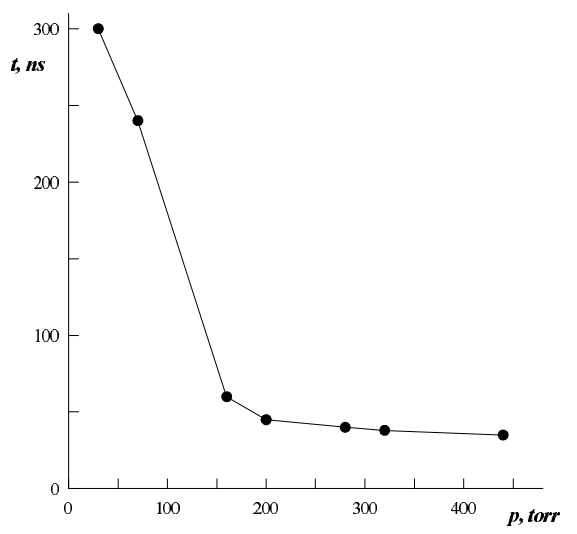

Fig. 8. Dependence of the stimulated emission pulse duration (FWHM) for $\mathrm{XeF}^{*}$ excimer molecule on the total pressure.

The same results were obtained for the $\mathrm{XeCl}^{*}$ and $\mathrm{KrF}^{*}$ excimer molecules at the excitation of $\mathrm{He}: \mathrm{Xe}(\mathrm{Kr}): \mathrm{HCl}\left(\mathrm{F}_{2}\right)$ mixtures by PTID. The temporal traces of the inductor voltage, spontaneous and stimulated emission pulses for $\mathrm{XeCl}^{*}$ (a) and $\mathrm{KrF}^{*}$ (b) excimer molecules are shown on Figure 9.

The maximum intensity of stimulated emission on the $308 \mathrm{~nm}\left(\mathrm{XeCl}^{*}\right)$ radiation wavelength was obtained for $\mathrm{He}: \mathrm{Xe}: \mathrm{HCl}$ - 96.8:3:0.2 mixture under the pressure of 120 torr and charging voltage $U_{c h}=26 \mathrm{kV}$ (Figure 9a). The pulse duration was approximately of $350 \mathrm{~ns}(\mathrm{FWHM})$. The results for $\mathrm{He}: \mathrm{Kr}: \mathrm{F}_{2}-89.8: 10: 0.2$ mixture at pressures of 92 and 200 torr with charging voltage $U_{c h}=27 \mathrm{kV}$ are shown on the Figure 9 (b). The maximum intensity of stimulated emission on the $248 \mathrm{~nm}\left(\mathrm{KrF}^{*}\right)$ radiation wavelength was obtained under the pressure of 200 torr. The pulse duration was about 80 ns (FWHM).

The gain factor measured using a cavity loss method for a maximum of a generation power of the excimer molecules described above was $0.01-0.015 \mathrm{~cm}^{-1}$.

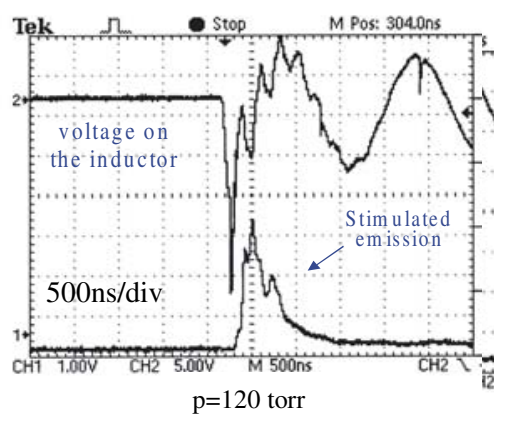

a)

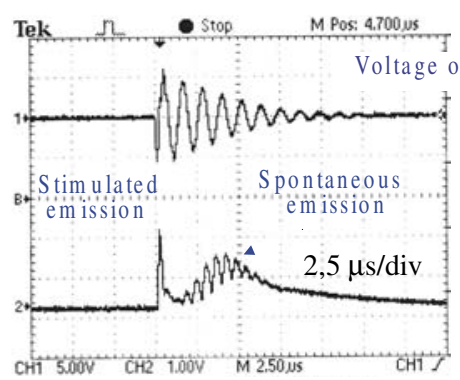

$\mathrm{p}=92$ torr

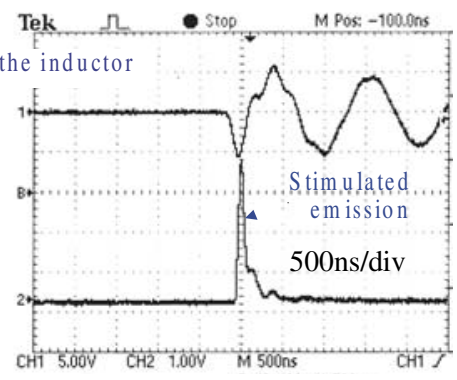

$\mathrm{p}=200$ torr

b)

Fig. 9. Temporal traces of the pulses of the voltage on the inductor, spontaneous and stimulated emission for $\mathrm{XeCl} *$ (a) and $\mathrm{KrF}^{*}$ (b) excimer molecules.

Proc. SPIE Vol. 6611, Laser Optics 2006: High-Power Gas Lasers, 66110G p. 1-11, April 2007 


\subsection{FI inductive laser}

Lasing on electronic transitions of fluorine atoms was obtained at excitation of the $\mathrm{He}: \mathrm{F}_{2}$ gas mixture under pressures in the range 20-1000 torr. Lasing was observed on eight lines in the wavelength range of 623-755 nm. The wavelengths and corresponding transitions are presented in the Table $1^{26}$.

Table 1. The wavelengths and its corresponding transitions of FI inductive laser.

\begin{tabular}{|c|c|c|}
\hline № & Wavelength, nm & Transition \\
\hline 1 & 623.97 & $3 \mathrm{p}^{4} \mathrm{~S}_{3 / 2}^{0} \rightarrow 3 \mathrm{~s}_{3 / 4}$ \\
\hline 2 & 634.85 & $3 \mathrm{p}^{4} \mathrm{~S}_{3 / 2}^{0} \rightarrow 3 \mathrm{~s}^{2} \mathrm{P}_{3 / 2}$ \\
\hline 3 & 641.36 & $3 \mathrm{p}^{4} \mathrm{~S}_{3 / 2}^{0} \rightarrow 3 \mathrm{~s}_{1 / 2}^{4}$ \\
\hline 4 & 703.75 & $3 \mathrm{p}^{2} \mathrm{P}_{3 / 2}^{0} \rightarrow 3 \mathrm{~s}^{2} \mathrm{P}_{3 / 2}$ \\
\hline 5 & 712.79 & $3 \mathrm{p}^{2} \mathrm{P}_{1 / 2}^{0} \rightarrow 3 \mathrm{~s}^{2} \mathrm{P}_{1 / 2}$ \\
\hline 6 & 731.1 & $3 \mathrm{p}^{2} \mathrm{~S}_{1 / 2}^{0} \rightarrow 3 \mathrm{~s}_{3 / 2}$ \\
\hline 7 & 739.87 & $3 \mathrm{p}^{4} \mathrm{P}_{5 / 2}^{0} \rightarrow 3 \mathrm{~s}_{5 / 2}^{4}$ \\
\hline 8 & 755.22 & $3 \mathrm{p}^{4} \mathrm{P}_{5 / 2}^{0} \rightarrow 3 \mathrm{~s}_{3 / 2}$ \\
\hline
\end{tabular}

It was found experimentally that the spectral structure and the lines intensity of the FI laser radiation strongly depend on $\mathrm{He}: \mathrm{F}_{2}$ ratio in the gas mixture and its total pressure. Figure 10 shows the dependence of the spectral structure and the lines intensity on the pressure in the range of 28 - 1000 torr at three different ratio of $\mathrm{He}: \mathrm{F}_{2}$ mixture.
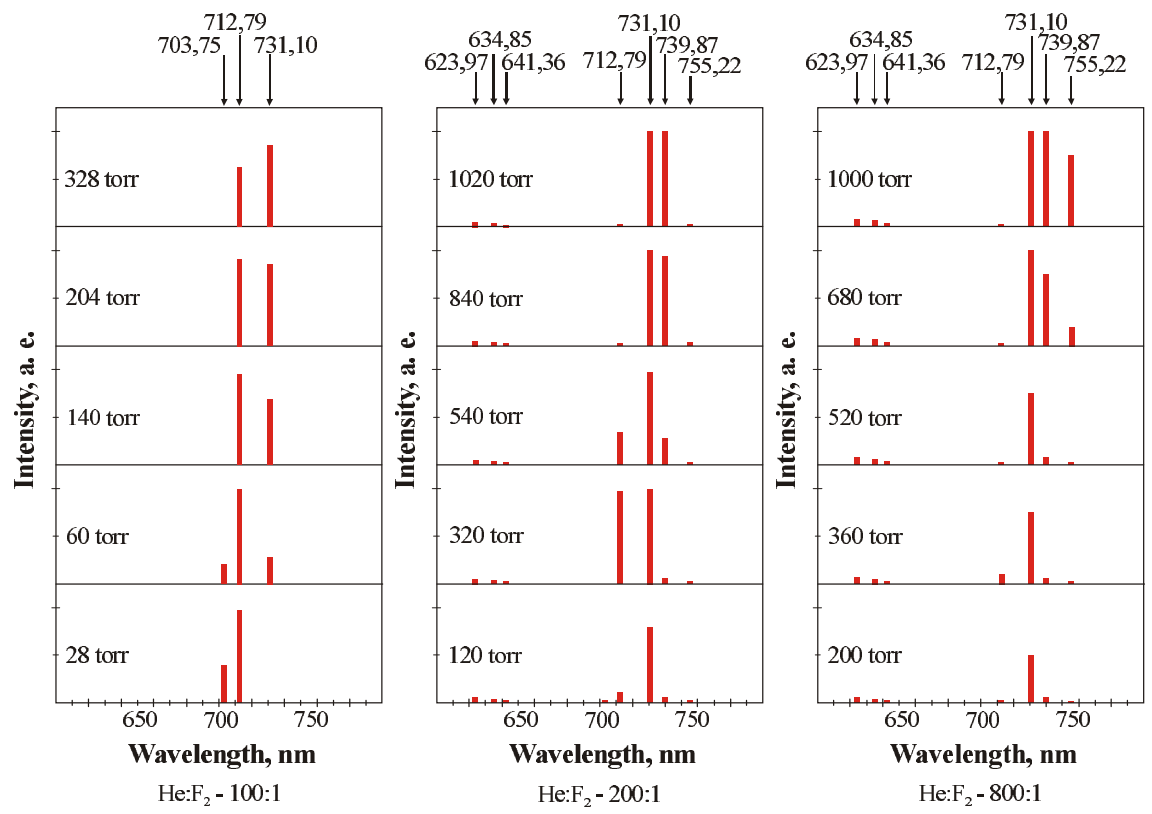

Fig. 10. Dependence of a spectral structure and lines intensity of the FI laser radiation on the total pressures for various He: $\mathrm{F}_{2}$ ratio of a gas mixture and.

The dependence of the radiation intensity on the ratio of the gas mixture components was investigated. The optimal composition for our excitation conditions was He: $\mathrm{F}_{2}-100: 1$ and the maximal laser radiation intensity was achieved at the pressure of 40 torr. In detail these investigations are described $\mathrm{in}^{25}$.On the Figure 11 the temporal traces of the inductor voltage and a laser pulse for the mixture $\mathrm{He}: \mathrm{F}_{2}-100: 1$ under a pressure of 40 torr at $27 \mathrm{kV}$ charging voltage are 
shown. The laser pulse appeared after $300 \mathrm{~ns}$ from the breakdown of the self-triggered spark gap $S G_{2}$ and it had the duration of $80 \pm 2$ ns (FWHM).

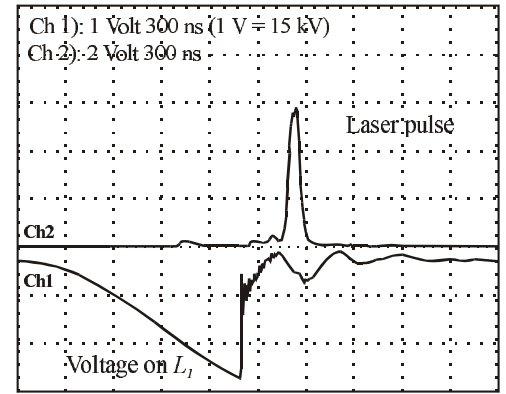

Fig. 11. Temporal traces of the inductor voltage $L_{l}$ and a laser pulse for the $\mathrm{He}: \mathrm{F}_{2}-100: 1$ mixture under a pressure of 40 torr.

Radiation was shaped in the form of a ring with outer diameter about $1.8 \mathrm{~cm}$ and an inner diameter of about $1.5 \mathrm{~cm}$ for discharge tube with inner diameter of $1.8 \mathrm{~cm}$ and a ring with outer diameter about $4.3 \mathrm{~cm}$ and an inner diameter of about $4.0 \mathrm{~cm}$ for discharge tube with inner diameter of $4.3 \mathrm{~cm}$. The measurement of laser emission divergence was performed by comparison of the laser beam near the laser window with one on the distance of $4.0 \mathrm{~m}$. As a result the divergence value was less than $10^{-4}$ radian.

The maximum output laser radiation energy was $0.85 \mathrm{~mJ}$ and it was approximately 7 times higher than that obtained in our previous paper ${ }^{25}$. The pulse power was $10 \mathrm{~kW}$ at the pulse duration of $80 \mathrm{~ns}$. The maximum gain measured using the cavity loss method was $0.025 \mathrm{~cm}^{-1}$.

\subsection{Nitrogen inductive laser at $337,1 \mathrm{~nm}$}

Lasing on electronic transitions of nitrogen molecules was obtained at an excitation of pure nitrogen gas under pressures in the range of $0.1-4.0$ torr by PTID. Lasing was observed on the $337,1 \mathrm{~nm}$ wavelength that corresponds to $(0,0)$ band of the $\mathrm{N}_{2}$ second positive system (Figure 12).

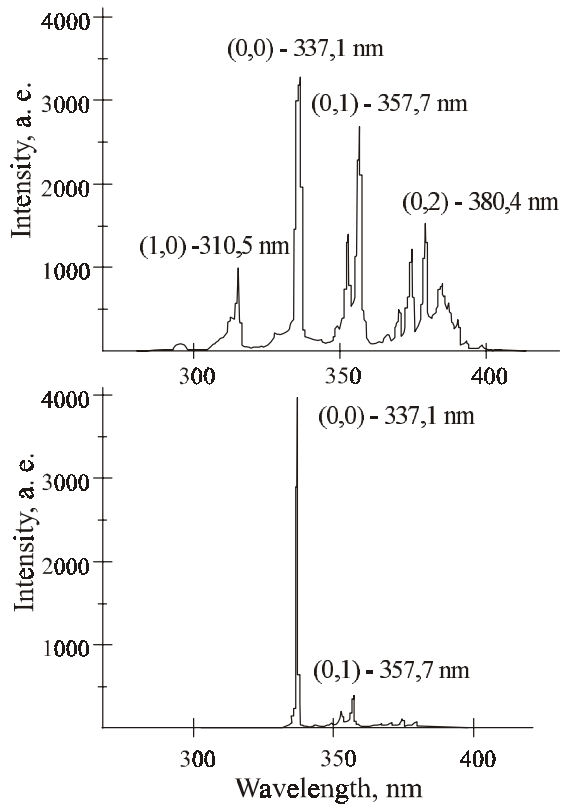

Fig. 12. Spectral lines of spontaneous (a) and laser (b) emission of the $N_{2}$ molecules. Charging voltage $U_{c h}=27 \mathrm{kV}$.

Proc. SPIE Vol. 6611, Laser Optics 2006: High-Power Gas Lasers, 66110G p. 1-11, April 2007 66110G-9 
On the Figure 13 the temporal traces of the inductor voltage and a laser pulse (a) and the detailed view of the laser pulse (b) for pure nitrogen under a pressure of 1.0 torr at $27 \mathrm{kV}$ charging voltage are shown. The laser pulse appeared with the breakdown of the self-triggered spark gap $S G_{2}$ (Figure 13a). The pulse duration (FWHM) was $13 \pm 0.5 \mathrm{~ns}$ (Figure 13b).

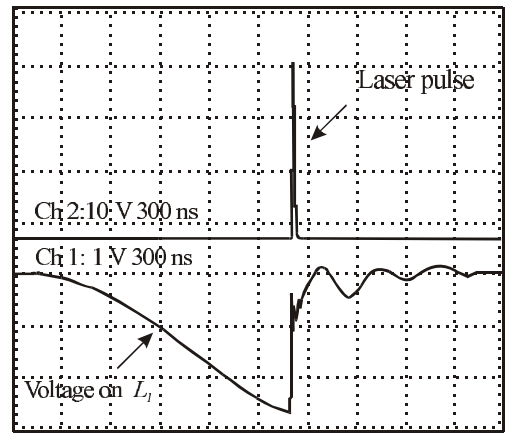

a)

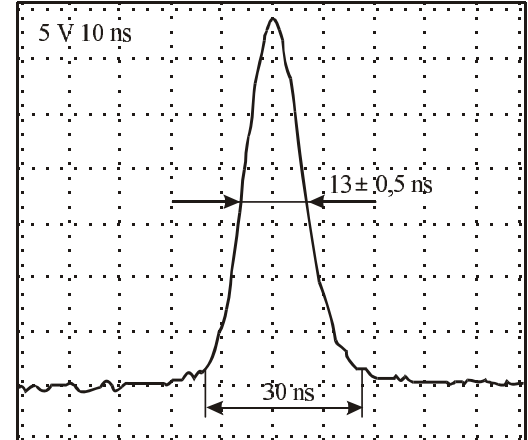

b)

Fig. 13. Temporal traces of the inductor voltage $L_{l}$ and a laser pulse $I$ (a) and detailed view of a laser pulse (b).

The laser beam has a ring form with dimension of outer diameter of $1.8 \mathrm{~cm}$ and inner one of $1.5 \mathrm{~cm}$ for a discharge tube with inner diameter of $1.8 \mathrm{~cm}$ and a ring with outer diameter about $4.3 \mathrm{~cm}$ and an inner diameter of about $4.0 \mathrm{~cm}$ for discharge tube with inner diameter of $4.3 \mathrm{~cm}$. For nitrogen inductive laser the divergence of laser emission on $337,1 \mathrm{~nm}$ wavelength was measured. The measurement was performed by comparison of the laser beam near the laser window with one on the distance of $10 \mathrm{~m}$. As a result the divergence value was less than $10^{-4}$ radian.

The maximum output laser radiation energy was $0.1 \mathrm{~mJ}$. At pulse duration of $13 \pm 0.5 \mathrm{~ns}$ (FWHM) this energy corresponds to a pulse power of $7.7 \mathrm{~kW}$. The maximum gain measured using the cavity loss method was $0.1 \mathrm{~cm}^{-1}$.

\section{CONCLUSION}

To achieve the lasing on the electronic transition of the atoms and molecules the excitation by the pulsed transverse inductive discharge was proposed. The excitation systems to obtain the pulsed inductive transverse discharge have been developed. The experimental study of spectral and temporal parameters of stimulated emission for active media on the $\mathrm{Ne}(585.3 \mathrm{~nm}), \mathrm{He}(587.5,667.8 \mathrm{~nm}), \operatorname{Ar}(750.4 \mathrm{~nm})$ atoms and $\mathrm{XeF}^{*}(353 \mathrm{~nm}), \mathrm{XeCl} *(308 \mathrm{~nm})$ and $\mathrm{KrF}^{*}(248 \mathrm{~nm})$, molecules excited by an inductive transverse discharge was performed.

For the first time the lasing on 8 lines of the fluorine (FI) atoms electronic transitions pumped by pulsed inductive transverse discharge was created. Output energy of $0.85 \mathrm{~mJ}$ was achieved.

The laser on the electronic transitions of the nitrogen molecules pumped by pulsed inductive transverse discharge was created. The laser action on the wavelength of $337.1 \mathrm{~nm}$ with output energy of $0.1 \mathrm{~mJ}$ at pulse power of $7.7 \mathrm{~kW}(13 \mathrm{~ns}$ at FWHM) was achieved.

\section{ACKNOWLEDGEMENTS}

The present work has been supported by Russian Foundation for Basic Research (Grant \# 06-02-16149-a).

\section{REFERENCES}

1. W.E. Bell, "Ring discharge excitation of gas ion lasers", Appl. Phys. Lett., 7, 7, 190-191, 1965.

2. J.P. Goldborough, E.B. Hodges, "RF induction excitation of cw visible laser transition in ionized gases", Appl. Phys. Lett., 8, 6, 137-139, 1966.

3. V.M. Mkhitaryan, "Lasers and radiation sources based on medium inductive discharge", Laser-Inform, №15-16, 18, 2004. (on Russian).

4. V.N. Ishchenko, V.N. Lisitsin, A.M. Razhev, 'Superradiative rare gas halide lasers excited by electric discharge”, Applied Physics, 12, 1, 55-58, 1977.

Proc. SPIE Vol. 6611, Laser Optics 2006: High-Power Gas Lasers, 66110G p. 1-11, April 2007 66110G-10 
5. V.N. Ishchenko, V.N. Lisitsin, A.M. Razhev, 'Efficient discharge pumping XeCl laser”, Optics Communications, 21, 1, 30-32, 1977.

6. P.L. Chapovsky, S.A. Kochubei, V.N. Lisitsin, A.M. Razhev, 'Excimer ArF/XeF lasers providing high -power stimulated radiation in Ar/Xe and F lines", Applied Physic s, 14, 2, 231-233, 1977.

7. A.M. Razhev, A.A. Zhupikov, E.S. Kargapol'tsev, "Excimer $\mathrm{KrCl}$ laser $(\lambda=223 \mathrm{~nm})$ on the mixture of $\mathrm{He}-\mathrm{Kr}$ HCl", Quantum Electron., 34, 2, 95-98, 2004.

8. A.M. Razhev, A.I. Shedrin, A. I. Kalyuzhnaya,A.V. Ryabtsev, A.A. Zhupikov, 'Effect of the pump intensity on the efficiency of a KrF excimer laser on a He-Kr-F2 mixture", Quantum Electron., 34, 10, 901-906, 2004.

9. F.V. Bunkin, V.I. Derzhiev, E.L. Latusch, I.I. Murav' iev, M.F. Sem, G.D. Chebotarev, S.I. Yakovlenko, A.M. Yancharina, 'Inversion and stimulated emiss ion due to a transition in NeI at $\lambda=585.3 \mathrm{~nm}$ in discharge with a "hard component", Quantum Electron., 16, 12, 1674-1676, 1986.

10. M.I. Lomaev, A.N. Panchenko, V.F. Tarasenko, "A study of the laser action in neon under pumping by a UV preionized self-sustained discharge", Quantum Electron., 17, 5, 631-634, 1987.

11. M.I. Lomaev, V.F. Tarasenko, 'Laser action in inert gases under pumping by a transverse discharge", Quantum Electron., 12, 10, 1237-1240, 1988.

12. M.I. Lomaev, V.F. Tarasenko, 'On the mechanism of inversio $n$ in $\mathrm{He}(\mathrm{Ne}, \mathrm{Ar})-\mathrm{NF}_{3}$ mixtures pumped by a selfsustained discharge”, Quantum Electron., 22, 2, 129-132, 1992.

13. M.A. Kovacs, C.J. Ultee, "Visible laser action in fluorine I", Appl. Phys. Lett., 17, 1, 39-40, 1970.

14. W.Q Jeffers, C.E. Wiswall, 'Laser action in atomic fluorine based on collisional dissociation of HF”, Appl. Phys. Lett., 17, 10, 444-447, 1970

15. A.E. Florin, R.J. Jensen, 'Pulsed laser oscillation at $0.7311 \mu$ from F atoms”, IEEE J. Quantum Electron. QE- 7, 2, 472-473, 1971.

16. J.R. English, III, H.C. Gardner, J.A. Merritt, 'Pulsed stimulated emission from N, C, Cl, and F atoms", IEEE J. Quantum Electron., QE-8, 11, 843-844, 1972.

17. D.G. Sutton, L. Galvan, P.R. Valenzuela, S.N. Suchard, "Atomic laser action in rare gas -SF 6 mixtures", IEEE J. Quantum Electron., QE- 11, 1, 54-57, 1975.

18. I.J. Bigio, R.F. Begley, 'High -power visible laser action in neutral atomic fluorine”, Appl. Phys. Lett., 28, 5, 263265, 1976.

19. L.O. Hocker, T.B. Phi, 'Pressure dependence of the atomic fluorine laser transition intensities", A ppl. Phys. Lett., 29, 8, 493-494, 1976.

20. T.R. Loree, R.C. Sze, 'The atomic fluorine laser: spectral pressure dependence", Optics Communications, 21, 2, 255-257, 1977.

21. V.N. Lisitsin, A.M. Razhev, 'Powerfull laser high -pressure on red lines of fluorine", JT Ph Letters, 3, 17, 862864, 1977. (on Russian).

22. L.O. Hocker, "High -resolution study of the helium-fluorine laser", J. Opt. Soc., Am. 68, 262-265, 1978.

23. S. Sumida, M. Obara, T. Fujioka, 'Novel neutral atomic lines in a high-pressure mixture of $F_{2}$ and He", J. Appl. Phys., 50, 6, 3884-3887, 1979.

24. J.E. Lawler, J.W. Parker, L.W. Anderson, W.A. Fitzsimmons, 'Experimental investigation of the atomic fluorine laser", IEEE J. Quantum. Electron., QE-15, 7, 609-613, 1979.

25. A.M. Razhev, V.M. Mkhitaryan, D.S. Churkin, "703 - to 731-nm FI laser excited by a transverse inductive discharge”, JETP Letters, 82, 5, 259-262, 2005.

26. A.R. Striganov, G.A. Odintsova., Tables of the spectral lines of atoms and ions, Handbook, Energoatomizdat, Moscow, 1982. (on Russian). 